\title{
Carbon C 11-JNJ-63779586
}

National Cancer Institute

\section{Source}

National Cancer Institute. Carbon C 11-JNJ-63779586. NCI Thesaurus. Code C148497.

A radioconjug ate composed of JNJ-63779586 labeled with the positron-emitting isotope carbon C 11, with potential use in imaging via positron emission tomography (PET). Upon administration of carbon C 11-JNJ-63779586, the JNJ-63779586 moiety acts as a substrate for the efflux transporter P-glycoprotein (P-gp) and the breast cancer resistance protein (BCRP). Upon PET imaging, this radiolig and may allow for the assessment of P-gp and BCRP function and expression in tumors. As P-gp and BCRP activity may influence response to therapy, measuring P-gp and BCRP activity may be beneficial when choosing chemotherapy. P-gp and BCRP, efflux transporters, are overexpressed by certain tumor cells and may contribute to multidrug resistance to chemotherapy. 